\title{
Corrigendum
}

\section{Improved detection reveals active $\beta$-papillomavirus infection in skin lesions from kidney transplant recipients}

Cinzia Borgogna, Simone Lanfredini, Alberto Peretti, Marco De Andrea, Elisa Zavattaro, Enrico Colombo, Marco Quaglia, Renzo Boldorini, Umberto Miglio, John Doorbar, Jan N Bouwes Bavinck, Koen D Quint, Maurits NC de Koning, Santo Landolfo and Marisa Gariglio

Modern Pathology (2014) 27, 917; doi:10.1038/modpathol.2014.2

Correction to: Modern Pathology advance online publication, 3 January 2014; doi: 10.1038/modpathol.2013.240

In this article, the funding sources were omitted from the Acknowledgment. A revised Acknowledgment is provided below.

\section{Acknowledgments}

We gratefully acknowledge 'Fondazione Banca Popolare di Novara per il territorio' for their contribution in purchasing the digital scanner Pannoramic
MIDI, and 'Stichting Pathologie Onderzoek en Ontwikkeling' for supporting the HPV genotyping. This work was supported by grants from Compagnia di San Paolo (CSP2012 to MG) and Associazione Italiana per la Ricerca sul Cancro-AIRC (IG 2012 n. 12850 to MG), ESCMID Research Grant 2013 (ESCMID 2013 to CB), and from the Ministry of Education, University and Research-MIUR (FIRB 2008 to MDA). The PhD Fellowships of Simone Lanfredini and Alberto Peretti are funded by Compagnia San Paolo and by 'Fondazione Franca Capurro per Novara Onlus', respectively. 